Mens

revue d'histoire intellectuelle de l'Amérique française

\title{
Aurélien Boivin, dir. Dictionnaire des œuvres littéraires du Québec, tome VII, 1981-1985. Saint-Laurent, Fides, 2003. 1229 p.
}

\section{Martine-Emmanuelle Lapointe}

Volume 5, numéro 2, printemps 2005

URI : https://id.erudit.org/iderudit/1024365ar

DOI : https://doi.org/10.7202/1024365ar

Aller au sommaire du numéro

Éditeur(s)

Centre de recherche en civilisation canadienne-française

ISSN

1492-8647 (imprimé)

1927-9299 (numérique)

Découvrir la revue

Citer ce compte rendu

Lapointe, M.-E. (2005). Compte rendu de [Aurélien Boivin, dir. Dictionnaire des œuvres littéraires du Québec, tome VII, 1981-1985. Saint-Laurent, Fides, 2003.

1229 p.l Mens, 5(2), 509-514. https://doi.org/10.7202/1024365ar d'utilisation que vous pouvez consulter en ligne.

https://apropos.erudit.org/fr/usagers/politique-dutilisation/ 


\section{Aurélien Boivin, dir. Dictionnaire des auvres littéraires du Québec, tome VII, 1981-1985. Saint- Laurent, Fides, 2003. 1229 p.}

Il apparait difficile de commenter un ouvrage aussi volumineux et exhaustif que le septième tome du Dictionnaire des cuvres littéraires du Québec. Comment rendre compte des différents points de vue que rassemble ce dictionnaire? Comment, en outre, éviter les généralisations abusives et les remarques ponctuelles ? Comme toute œuvre collective de long souffle, le Dictionnaire des cuvres littéraires du Québec (1981-1985) regroupe un nombre impressionnant de chercheurs, étudiants et professeurs, qui ont bien voulu se prêter au jeu de la synthèse et de l'érudition. L'ouvrage offre une collection de lectures, divergentes, variées, nécessairement fragmentaires, qui ne peuvent être facilement résumées et commentés.

Respectant les lois du genre auquel il ressortit, le DOLQ fournit une vue d'ensemble, voire un tableau relativement achevé, de la période étudiée. Fort bien conçu, il comprend une introduction, une chronologie et une bibliographie générales. Recensées par ordre alphabétique, les entrées portent sur des œuvres, des objets culturels ou des compagnies théâtrales. Elles sont le plus souvent accompagnées de bibliographies, permettant ainsi au lecteur d'approfondir sa connaissance des ouvres et des auteurs retenus. Cela dit, les œuvres recensées ne sont pas toutes d'égale qualité et ne sauraient par là même inspirer des lectures toujours aussi riches et stimulantes. Les textes marquants de la période sont souvent analysés en profondeur tandis que les textes mineurs, ceux que la critique journalistique et universitaire a parfois négligés avec raison, sont rapidement commentés. Il est certes plus inspirant de s'attarder à Vie et mort du roi boiteux de Jean-Pierre Ronfard, à Agonie de Jacques Brault ou à La détresse et l'enchantement de Gabrielle Roy qu'à certains ouvrages obscurs que la 
postérité n'a pas retenus. Outil néanmoins indispensable pour quiconque s'intéresse un tant soit peu à la littérature québécoise des années 1981-1985, le septième tome du DOLQ possède les défauts et les qualités d'un ouvrage dont l'ambition première demeure l'exhaustivité.

Parmi les contraintes de l'ouvrage, retenons la brièveté de la période étudiée. Marquées par une pluralité de styles, par " une égalité des voix " selon l'hypothèse fort convaincante d'André Brochu, dominées par l'institutionnalisation grandissante du milieu culturel - en témoignent la création de nouveaux prix et l'attribution de plus en plus systématique de subventions à l'édition - les années 1981-1985 ont vu paraitre un nombre considérable de romans (plus de 400 selon les auteurs de l'introduction), de recueils poétiques et d'essais, cela sans oublier la diffusion de nombreux spectacles théâtraux. C'est sans doute pour cette raison que les auteurs du DOLQ n'ont guère tenté d'attribuer une cohérence surfaite à une production littéraire et culturelle aussi diversifiée. Ils ont également évité, rompant ainsi avec la perspective critique privilégiée dans les précédents tomes (voir le tome IV notamment consacré aux œuvres des années 1960-1969), de rattacher trop étroitement le domaine culturel aux enjeux politiques et sociaux qui ont dominé la vie publique des années 1981-1985.

L'une des difficultés inhérentes à la composition d'un si vaste ouvrage est sans doute la définition de l'objet d'étude. Or, la littérature québécoise, qui semblait naguère correspondre au projet d'un groupe culturel clairement identifié, connaît une réinterprétation à l'époque contemporaine. En témoignent notamment les travaux de Pierre Nepveu (L'écologie $d u$ réel, 1988), ceux des auteurs de l'ouvrage collectif Fictions de lidentitaire au Québec (1991) et les réflexions des chercheurs réunis par le groupe de recherche Montréal imaginaire. Les di- 
recteurs du DOLQ ont d'ailleurs assoupli leur définition de la littérature québécoise en incluant les spectacles théâtraux à leur septième tome, initiative tout à fait louable qui permet de mesurer les interactions entre la dramaturgie, les mises en scène et les explorations des différentes troupes présentes sur la scène théâtrale de l'époque. Néanmoins, les critères retenus dans l'avertissement de l'ouvrage ne s'avèrent pas toujours convaincants. Pour être considérée "québécoise », une œuvre doit « avoir été éditée par une maison québécoise ; avoir été écrite par un Québécois/une Québécoise ou par une personne ayant décidé de vivre au Québec; viser le Québec comme lieu de consécration; relever, en tout ou en partie, de l'imaginaire ou du réel québécois. Une œuvre québécoise, sauf exception, doit répondre à au moins deux de ces critères ». Si les deux premiers critères semblent aller de soi, les deux derniers paraissent plutôt vagues et peuvent facilement prêter à confusion. Que signifie au juste « relever, en tout ou en partie, de l'imaginaire ou du réel québécois " ? Cela renvoie-t-il à la matière même des œuvres, à leurs lieux d'inspiration ou cela désigne-t-il le fait d'appartenir à la mémoire collective québécoise? Par ailleurs, pourquoi ne pas avoir ajouté le critère suivant: doit être écrite en langue française. On le constate au fil de la lecture, il s'agit d'un impératif tacite puisque toutes les œuvres recensées sont de langue française. Pourtant, la littérature anglo-québécoise, qui est écrite par des Québécois(es) et relève de l'imaginaire culturel du Québec, n'est guère abordée. Malencontreux oubli, réflexe de lecteurs trop familiers avec une définition stricte de la littérature québécoise, on ne sait trop? Mais il n'en demeure pas moins que les ouvres de David Solway, Clark Blaise, David Fennario, Gail Scott et Hugh Hood, pour ne nommer que celles-là, appartiennent elles aussi à la littérature québécoise. Afin de dissiper les malentendus, les auteurs auraient pu fournir une dé- 
finition plus stricte et plus claire de leur objet d'étude, lequel ne renvoie peut-être pas de manière aussi évidente à la production littéraire et culturelle d'un groupe linguistique homogène.

Dans l'introduction de l'ouvrage, rédigée par Aurélien Boivin, Roger Chamberland, Gilles Dorion et Gilles Girard, le lecteur trouvera une vaste synthèse situant les œuvres et leurs auteurs dans le champ littéraire québécois. Les principaux genres, soit le roman, la poésie, le théâtre, l'essai et les formes autobiographiques constituent les grandes divisions de cette introduction qui accorde une large place au contexte institutionnel et aux nouvelles thématiques explorées par les auteurs de la période. La partie consacrée au roman s'avère beaucoup plus étoffée que les autres sections et semble ainsi confirmer l'hégémonie du genre sur les autres formes. Les auteurs y multiplient cependant les thèmes et les sous-catégories génériques, ce qui a parfois pour effet de confondre le lecteur. Comment distinguer par exemple, le « roman historique » et le "roman social» ? Les auteurs évoquent le "roman historique ou à caractère historique » (p. xx) sans en préciser davantage les contours. Cette indécision terminologique conduit parfois à des catégorisations approximatives. Pourquoi attribuer un caractère historique à la trilogie d'Alice Parizeau, mais inclure les Chroniques du Plateau Mont-Royal de Michel Tremblay dans la catégorie du roman social ? Alice Parizeau comme Michel Tremblay traitent dans leurs œuvres des années 1940 et tentent d'en reconstituer l'horizon social et référentiel. En outre, un certain flou sémantique entoure le thème de la quête identitaire : "plusieurs romans de la période, écriton, s'intéressent à la quête identitaire, qui s'accompagne souvent d'une fuite vers un ailleurs où le personnage principal espère trouver le bonheur qui lui est refusé dans le monde qu'il habite et dans lequel il se sent menacé » (p. xxiii). Il s'agit 
là certes d'un scénario récurrent, commun à plusieurs des romans parus entre 1981 et 1985 . Le thème constitue toutefois une catégorie fourre-tout, plus commode qu'opératoire, renvoyant aussi bien au désir d'une libération, à la prise de parole qu'à la quête du bonheur, du père ou du pays.

Les parties portant sur la poésie, le théâtre et l'essai apparaissent plus éloquentes, dans la mesure où les regroupements, sans doute moins artificiels, y permettent de mieux cerner les courants, aussi diffus et insaisissables soient-ils, qui ont traversé la production littéraire québécoise de la période. Dans la partie sur la poésie, les auteurs évoquent avec raison l'effacement des préoccupations formalistes partagées par plusieurs poètes des années 1970. Dans la partie consacrée aux spectacles théâtraux et à la dramaturgie, ils montrent bien comment le «théâtre de recherche, marqué par les explorations de plusieurs troupes toujours bien vivantes aujourd'hui - Carbone 14, Omnibus, le Théâtre Repère notamment a privilégié la création multidisciplinaire et «les possibilités expressives de la voix et du corps » (p. xxxvi). On pourra cependant reprocher aux auteurs leurs partis pris souvent trop clairement affichés. Pourquoi par exemple qualifier le théâtre réaliste de "carcan» (p. xxxvi) ? Pourquoi égratigner au passage le textocentrisme, le théâtre engagé et les thématiques nationalistes explorées par les dramaturges des années 1960 1970 ? Ces jugements implicites laissent entendre que la pratique théâtrale a connu une sorte d'évolution positive et reconduisent souterrainement une lecture téléologique de l'histoire littéraire.

La section consacrée au genre de l'essai s'avère malheureusement très brève. Plusieurs essais littéraires et recueils de textes critiques parus au cours de la période ont pourtant marqué l'histoire de la littérature québécoise, qu'il s'agisse d'Y a-t-il un intellectuel dans la salle? d'André Belleau, des Trois 
essais sur l'insignifiance de Pierre Vadeboncœur, de La littérature contre elle-même de François Ricard ou de L'échappée des discours de l'cil de Madeleine Ouellette-Michalska. Si les auteurs évoquent la " "diversité" de cette catégorie "élastique" " qu'est l'essai (p. xli), ils accordent peu d'importance aux différents discours culturels qui ont pu nourrir la pratique des essayistes des années 1981-1985 et aux revues - Liberté, Vice Versa, La Gazette des femmes entre autres - qui demeurent des lieux de réflexion essentiels à la diffusion du genre.

En évoquant ici, et en toute subjectivité, les limites et les failles de ce septième tome du DOLQ, je ne voudrais surtout pas en décourager la lecture. L'ouvrage constitue un bilan non négligeable et respecte en cela le mandat qui lui était attribué. Voué à fournir des textes introductifs aux œuvres, des survols, il contribue à enrichir la littérature critique, encore timide et modeste, portant sur la période des années 19811985. Ne prétendant nullement renouveler la réflexion sur les œuvres, ne proposant pas d'envolées interprétatives ni de grandes intuitions théoriques, il se présente à la fois telle une borne, voire un livre-repère, et une synthèse.

Martine-Emmanuelle Lapointe Département de langue et littérature françaises Université McGill

Guy Laflèche et Serge Trudel. Un janséniste en Nouvelle-France. Laval, Éditions du Singulier, 2003. 318 p.

Voici un ouvrage intrigant, dont le but premier est de remettre en question la paternité d'un ouvrage datant de l'époque de la Nouvelle-France, soit le Premier Établissement de la 